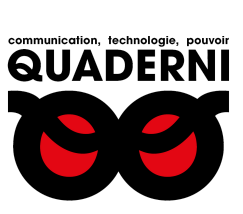

\title{
Quaderni
}

Communication, technologies, pouvoir

\section{L'État se meurt. Un méta-diagnostic comparé de la métropolisation en France et aux États-Unis}

Cynthia Ghorra-Gobin, La métropolisation en question

\section{Claire Tollis}

\section{OpenEdition}

\section{Journals}

Édition électronique

URL : https://journals.openedition.org/quaderni/1110

DOI : 10.4000/quaderni.1110

ISSN : 2105-2956

Éditeur

Les éditions de la Maison des sciences de l'Homme

Édition imprimée

Date de publication : 5 octobre 2017

Pagination : 91-96

\section{Référence électronique}

Claire Tollis, «L'État se meurt. Un méta-diagnostic comparé de la métropolisation en France et aux États-Unis », Quaderni [En ligne], 94 | Automne 2017, mis en ligne le 05 octobre 2017, consulté le 23 juillet 2021. URL : http://journals.openedition.org/quaderni/1110 ; DOI : https://doi.org/10.4000/ quaderni. 1110 


\section{Compte rendu}

\section{L'État se meurt \\ Un méta-diagnostic comparé de la métropolisation en France et aux États-Unis}

par Claire Tollis

Université d'Artois - Discontinuités

Laboratoire TVES

\section{À propos de l'ouvrage, La métropolisation en question}

\section{Cynthia Ghorra-Gobin}

PUF, 2015

1. II n'est pas évident de les citer de manière exhaustive mais nous pensons notamment à La Métropole parisienne (Saint Julien, Le Goix, 2007), Paris, alchimie d'une métropole (Baudouin \& al., 2008), La Métropole (Rafele, 2010), Le Temps de la Métropole, ( VermeyIen, 2014), La Métropole Logistique (Dablanc,

Frémont, 2015), Grenoble, le pari de la métropole (Lajarge, Bloch, 2015), La Métropole par le projet (Collectif, 2016).
Le roi : Je meurs, je meurs.

Marie : Tu as préparé tout cela.

Le roi : Sans le faire exprès.

Le roi se meurt, Eugène Ionesco, 1962.

Dans le flot actuel des travaux universitaires sur la métropole ${ }^{1}$, le livre de Cynthia Ghorra-Gobin, La métropolisation en question se distingue par son ambition comparatiste et critique, ainsi que par son approche processuelle et multiscalaire. L'auteure explique ainsi, avec une grande limpidité, comment s'est construite la dynamique institutionnelle et spatiale de métropolisation et les effets qui en résultent dans chacune des deux aires étudiées (France/ États-Unis) : principalement, des configurations problématiques des territoires et un affaiblissement de l'État.

\section{Que devient l'État à l'heure de la métropolisation consentie ?}

L'ouvrage est court et efficace (110 pages). Il est paru fin 2015 dans la collection «La ville en débat » des Presses Universitaires de France. On peut regretter le titre trop timide au regard des enjeux soulevés et discutés dans le 
livre. Cynthia Ghorra-Gobin le souligne dès l'introduction : son opus paraît à un moment charnière, l'institutionnalisation en France de la métropole comme échelle de planification de la ville constitue une «opportunité de discuter des enjeux que représente la question métropolitaine pour l'ensemble des habitants comme pour l'État ». C'est là le double angle d'attaque de l'auteure : que deviennent les habitants ? Que va/doit devenir l'État ? Ces questionnements sont alimentés par l'assimilation et la mise en dialogue de travaux universitaires français et anglo-américains et abordés à travers une analyse fine des acteurs de l'aménagement, des textes qu'ils produisent (lois, normes, rapports) et la restitution de quelques instantanés de vie, c'est-àdire de situations incarnant les problématiques en jeu et à même de dire des choses sur le vécu du phénomène.

L'ouvrage repose donc sur l'exploitation d'un matériau sur mesure, à même de capter le (méta)discours des institutions sur ce qu'elles sont en train de produire en termes de formes urbaines mais aussi d'inégalités, de tensions et d'incompréhensions. Cynthia Ghorra-Gobin nous livre une photographie panoramique et évolutive de ces manifestations, complétée par des effets de zoom sur certains problèmes (les contraintes de mobilité des populations les plus pauvres, la perte culturelle de repères d'identification territoriale, les dépenses abusives liées au millefeuille décisionnel et administratif, notamment). Il s'agit finalement d'un « méta-diagnostic » du phénomène métropolitain : une analyse globale et détaillée de son processus d'émergence, de sa très probable consolidation et de ses limites.

\section{L'État dépassé : la métropolisation aux États-Unis, un phénomène surtout économique?}

Le médecin : Cela fond, cela s'évapore,

il n'en reste pas une goutte,

pas une poussière,

pas une ombre.

Le roi se meurt, Eugène Ionesco, 1962.

Le premier chapitre met en avant, dans le cas des États-Unis, comment la subversion économique des territoires métropolitains liée aux mutations du capitalisme globalisé a supplanté l'anticipation des autorités publiques à une échelle nationale, pour donner à voir une mutation fonctionnelle de 
2. M. Orfield, American Metropolitics. The New Suburban Reality, Washington DC, Brookings Institution Press, 2002. l'organisation des villes et de leurs périphéries. Le dualisme ville-périphérie étant dépassé et remplacé par une pluralité d'expressions, l'auteure explore les mots qui décrivent cette self-made métropole et ceux qui émergent pour tenter de cadrer son devenir.

Au départ, le phénomène est surtout économique mais il devient politique de par ses enjeux socio-spatiaux : la métropole constitue un terrain d'opportunité, de placement, une vitrine politicienne. Les métropoles aujourd'hui récupérées comme « locomotives de l'économie nationale» ne sont plus livrées à la seule main invisible, elles bénéficient d'investissements de la part de l'État fédéral, des États fédéraux et des gouvernements municipaux : elles sont entrées dans les classements statistiques renseignant la compétitivité territoriale et les agendas politiques. L'auteure explique que de grands espoirs sont placés dans les metropolitics (Orfield $\left.{ }^{2}, 2002\right)$, pour contrer les effets ou les non-effets des broken politics de l'État fédéral, dépassé.

\section{L'État surmené : la métropolisation à la française, entre volontarisme et déni}

Marie, se précipitant vers le roi : Mon Roi, vous boitez.

Le roi, faisant deux ou trois pas en boitant légèrement :

Je boite? Je ne boite pas. Je boite un peu.

Le roi se meurt, Eugène Ionesco, 1962

Le deuxième chapitre montre, dans le cas français, comment le volontarisme politique lié à la décentralisation et l'aide attribuée par l'État aux grandes villes - pour les faire rayonner dans le même contexte de capitalisme/globalisation - ont renforcé le pouvoir des agglomérations, soutenant le principe de proximité positive et contribuant à un étalement urbain aujourd'hui paroxystique. La métropole est donc planifiée dans le cas français. Malheureusement, l'auteure met en avant combien décentraliser ne revient pas forcément à équilibrer.

Le premier mouvement de décentralisation/inter-communalisation est particulièrement critiqué par $\mathrm{C}$. Ghorra-Gobin : les coopérations mises en place à l'échelle locale sont avant tout affinitaires, ce qui participe à un émiettement intercommunal nuisant à l'attractivité territoriale et génère un millefeuille administratif dépensier. 
Quelques lois nationales viennent bien affirmer dans les années 2000, le droit à l'expérimentation et une certaine autonomie financière pour les collectivités territoriales. Toutefois, la stratégie de Lisbonne, effort collectif planifié par le Conseil Européen pour adapter l'Europe à la mondialisation (2000-2010) met en avant le retard de la France dans la valorisation des métropoles, ce qui montre un décalage entre ce que l'État dit et ce qu'il fait.

Pollution, étalement urbain, ségrégation sont mis en avant dans les analyses et un autre problème surgit, celui des villes moyennes, oubliées de cette planification des territoires.

Déclarées mais non assumées politiquement et non accompagnées financièrement par l'État, décentralisation et intercommunalité n'aboutissent pas. L'État français s'essouffle, en vain. Dans ce contexte, l'institutionnalisation de la métropole apparaît nécessaire. Elle serait même pressentie comme remède pour endiguer une certaine «souffrance spatiale ».

En 2014, la loi MAPTAM (Modernisation de l'Action Publique Territoriale et d'Affirmation des Métropoles) crée des métropoles dotées désormais de budgets plus importants et prévoyant que les membres des conseils métropolitains soient élus au suffrage universel dès 2020, cette loi vise les territoires comme ferments de la croissance. En instillant « confiance, clarté, cohérence, démocratie », l'État français entend redresser la France en partant des territoires. Il semble évident que la métropole d'aujourd'hui se distingue d'un « simple outil d'aménagement » tel qu'envisagé dans les années 1960. Ceci dit, il demeure une ambiguïté : l'État veut décentraliser, donc lâcher certaines prérogatives, mais sans lâcher trop de son pouvoir (conditionnement des aides, contrôle, veille). En dédoublant ses missions sans n'en céder vraiment aucune, l'État se retrouve en situation de surchauffe, proche du burn-out. Comment, dans ce contexte, envisager une économie de moyens ? Comment envisager sans absurdité que la démocratie participative locale ait un sens quand tout (ou presque) reste conditionné par un État surmené ?

\section{L'État se meurt : l'utopique égalité des territoires}

Le roi : Vous les suicidés, apprenez-moi, comment il

faut faire pour acquérir le dégoût de l'existence.

Apprenez-moi la lassitude. 
3. Hank V. Savitch,

" Globalisation et changement d'échelle dans le gouvernement urbain », revue Métropole $\mathrm{n}^{\circ} 2$, pp. 133-166, 2007.
Quelle drogue faut-il prendre pour cela?

Le roi se meurt, Eugène Ionesco, 1962.

Le troisième chapitre constitue vraiment un défi : l'auteure met en perspective les dynamiques étudiées et les enjeux soulevés dans les deux pays. Elle y élabore une critique construite, nuancée et percutante de l'État tel qu'il se meurt aujourd'hui. Dans le cas français, comme dans le cas états-unien, la mission principale attribuée à l'État est d'encourager ce que les territoires ne sont a priori pas capables d'assurer : l'égalité. Seulement, ils n'y parviennent pas.

En zoomant sur des problématiques territoriales (les précaires de la mobilité, et plus largement les ségrégations), résultant des configurations spatiales issues de la métropolisation (cette fameuse glocalité), Cynthia Ghorra-Gobin explique en quoi le paradigme métropolitain échoue à enrayer la polémique. Si la question physique et matérielle des périmètres (ou d'une géométrie variable) se pose, c'est bien la question de nouveaux assemblages de droits et d'autorités qui est soulevée par l'auteure. « À la dynamique centripète de l'État, se substituerait une multiplication d'assemblages territoriaux de nature centrifuge » (p. 89). La question n'est donc pas, d'après l'auteure, de recomposer le territoire mais bien de reconfigurer l'État, ses formes d'intervention et ses compétences.

L'auteure prend ici fermement parti : l'État doit changer. Son injonction très argumentée pour le rééchelonnement des pouvoirs s'appuie sur quatre modèles de gouvernance métropolitaine (inspirés de la pensée de H. Savitch ${ }^{3}$, 2007) illustrés par des exemples concrets d'organisation (Indianapolis, Portland, Los Angeles, Lille).

L'État serait appelé (par C. Ghorra-Gobin mais d'abord par d'autres chercheurs comme Brenner, Swyngedouw ou encore Pinson \& Le Galès) à se réinventer, ses fonctions étant déplacées à un niveau supranational (Union Européenne / Alena), ainsi qu'à l'échelle infranationale (les métropoles). L'État aurait pour mission de donner du sens aux relations entre ces échelles et entre les territoires (notamment, entre les métropoles, mais aussi entre les métropoles et les territoires qui n'en font pas partie), en reconnaissant la métropole comme partenaire plus que comme un simple exécutant. C'est à cette échelle que l'auteure, inspirée par les travaux d'Iris Marion Young, entrevoit la possibilité d'une prise en charge de la justice spatiale et sociale, 
c'est-à-dire d'une solidarité territoriale possible entre quartiers des cités et quartiers gentrifiés, à l'heure du « branchage » mondialisé.

\section{Tout s'arrange...?}

Le roi : Qu'avez-vous tous à me regarder ainsi?

Est-ce qu'il y a quelque chose d'anormal?

Il n'y a plus rien d'anormal

puisque l'anormal est devenu habituel.

Ainsi, tout s'arrange.

Le roi se meurt, Eugène Ionesco, 1962.

Pour conclure, Cynthia Ghorra-Gobin affirme que les métropoles sont devenues des lieux de pouvoirs mais non des lieux d'identification. Si l'échelle est pertinente pour l'action publique en termes de gestion du foncier et d'observation des loyers, en termes d'évaluation des vulnérabilités, il manque un grand récit contemporain qui soit fédérateur pour les habitants.

Il convient ici de créditer l'auteure d'une parfaite clarté dans son exposé. Son ouvrage constitue à la fois une excellente synthèse des travaux récents sur la métropole en France et aux États-Unis et un diagnostic holiste des problématiques concernant les configurations spatiales et politiques en train de se construire. La connaissance comparée de l'avènement des métropoles permet de mieux en comprendre les enjeux contemporains. Enfin, si l'approche critique va crescendo, l'on peut regretter qu'elle ne soit pas plus affirmée dès l'introduction et surtout qu'elle s'essouffle au moment de la conclusion : de quoi l'État va-t-il bien pouvoir démissionner (vraiment)? 